\title{
ABT-263, a Bcl-2 inhibitor, enhances the susceptibility of lung adenocarcinoma cells treated with Src inhibitors to anoikis
}

\author{
YUJI SAKUMA, JUN TSUNEZUMI, YOSHIYASU NAKAMURA, MITSUYO YOSHIHARA, \\ SHOICHI MATSUKUMA, SHIRO KOIZUME and YOHEI MIYAGI
}

Molecular Pathology and Genetics Division, Kanagawa Cancer Center Research Institute, Yokohama, Japan

Received October 28, 2010; Accepted December 2, 2010

DOI: $10.3892 /$ or.2010.1123

\begin{abstract}
The tyrosine kinase Src plays an important role in the development of anoikis resistance in lung adenocarcinomas. Several suspension lung adenocarcinoma cell lines, which express phosphorylated Src, undergo apoptosis, or anoikis, in the presence of Src kinase inhibitors. However, lung adenocarcinoma cell lines vary in their sensitivity to Src inhibitors. We hypothesized that the addition of ABT-263, a potent Bcl-2 inhibitor, should significantly enhance the degree of anoikis in lung adenocarcinoma cells treated with Src inhibitors. In this study, we treated four suspension lung adenocarcinoma cell lines with ABT-263, an Src inhibitor (bosutinib or PP1), or a combination of both. In LC-KJ and HCC827 cells, combined treatment with ABT-263 and an Src inhibitor effectively induced anoikis, and the extent of anoikis was significantly greater than that induced by each agent alone; the synergy between the two drugs was apparent. Although we did not observe a marked increase in anoikis in LC-KJ and HCC827 cells treated with ABT-263 alone, H1650 and H1975 cells treated with ABT-263 (1 $\mu \mathrm{M})$ upon detachment significantly underwent apoptosis, the levels of which were much greater than those observed upon attachment. However, the levels of anoikis induced by combination treatment were still greater than those by the individual agents in H1650 and H1975 cells. These findings provide a biological rationale to test combination therapy with ABT-263 and Src inhibitors in patients with lung adenocarcinoma.
\end{abstract}

\section{Introduction}

We previously reported that approximately half of patients with a small $(\leq 2 \mathrm{~cm}$ in size) lung adenocarcinoma with vessel invasion experience relapse in the form of distant metastasis, even after complete surgical tumor resection (1).

Correspondence to: Dr Yuji Sakuma, Molecular Pathology and Genetics Division, Kanagawa Cancer Center Research Institute, 1-1-2 Nakao, Asahi-ku, Yokohama 241-0815, Japan

E-mail: ysakuma@gancen.asahi.yokohama.jp

Key words: lung adenocarcinoma, anoikis resistance, ABT-263, $\mathrm{Src}$
These clinicopathological data indicate that lung adenocarcinoma cells floating in vessels do not undergo apoptosis. In other words, the cells have acquired the ability to resist anoikis, a type of apoptosis induced by detachment from their extracellular matrix (ECM) (2). Several reports have shown that Src, a non-receptor type tyrosine kinase, plays an important role in the anoikis resistance in lung adenocarcinomas (3-5). We have recently demonstrated that i) intralymphatic floating lung adenocarcinoma cells observed in primary tumor tissues form nests expressing E-cadherin and the amount of phosphorylated Src expressed by the floating cells is much greater than that of ECM-adhesive tumor cells; ii) Src is induced and phosphorylated in tumor spheroids of four suspension lung adenocarcinoma cell lines (LC-KJ, HCC827, H1650 and H1975), the shape of which is similar to intralymphatic tumor cell nests observed in tissues; and iii) the four types of spheroids undergo anoikis to varying degrees in response to treatment with Src kinase inhibitors (5). However, not all four lung adenocarcinoma cell lines treated with Src inhibitors readily or uniformly underwent anoikis (5). These results prompted us to search for drugs that can significantly enhance the susceptibility of lung adenocarcinoma cells treated with Src inhibitors to anoikis.

Apoptosis occurs through the death receptor (the extrinsic pathway) or Bcl-2-regulated (the intrinsic or mitochondrial) pathways. Most drugs, as well as growth factor withdrawal, induce apoptosis by activation of the intrinsic pathway $(6,7)$. The Bcl-2 family of proteins controls the integrity of the outer mitochondrial membrane (OMM) and plays a critical role in determining the susceptibility of cells to apoptosis via the intrinsic pathway. The Bcl-2 family comprises antiapoptotic members such as Bcl-2, Bcl- $\mathrm{X}_{\mathrm{L}}$, Bcl-w and Mcl-1, pro-apoptotic members such as Bax and Bax, and the proapoptotic Bcl-2 homology domain (BH) 3-only proteins such as Bim, Bad and Puma (6,7). The relative balance between the anti-apoptotic members and pro-apoptotic $\mathrm{BH} 3$-only proteins usually defines commitment to apoptosis (8). BH3only proteins bind the anti-apoptotic members to relieve their inhibition of Bax and Bak resulting in oligomerization and activation of Bax and Bak, which leads to OMM permeabilization, release of cytochrome $\mathrm{C}$ into the cytoplasm, and caspase activation (6-8). ABT-737, a small-molecule inhibitor of Bcl-2, Bcl- $\mathrm{X}_{\mathrm{L}}$ and $\mathrm{Bcl}-\mathrm{w}$, and its orally bioavailable derivative, ABT-263, exhibit almost identical function to the 
BH3-only protein Bad, and are referred to as Bcl-2 inhibitors or BH3 mimetics $(9,10)$. Among lung carcinomas, most small cell carcinomas have been confirmed to be highly sensitive to ABT-737 (or ABT-263), whereas non-small cell lung carcinomas (NSCLCs), including adenocarcinomas, seem to be relatively resistant to Bcl-2 inhibitors $(9,10)$. It remains unknown whether suspension lung adenocarcinoma cells cultured undergo anoikis in response to treatment with Bcl-2 inhibitors. It has been reported that ABT-737 (or ABT263) display synergistic cytotoxicity with chemotherapeutics and radiation $(6,7,9,10)$. Furthermore, in several lung adenocarcinoma cell lines, epidermal growth factor receptor (EGFR) kinase inhibitor (gefitinib or erlotinib)-induced apoptosis is increased by combination treatment with ABT$737(11,12)$. However, the efficacy of combination therapy with Src kinase inhibitors and ABT-737 (or ABT-263) remains to be clarified. Since the BH3-only protein Bim was markedly induced in lung adenocarcinoma cells upon detachment when treated with Src inhibitors (5), we hypothesized that the addition of ABT-263 should enhance the degree of anoikis in lung adenocarcinoma cells treated with Src inhibitors. The objective of the present study was to elucidate to what degree four types of suspension lung adenocarcinoma cell lines cultured undergo anoikis in the presence of ABT-263, Src inhibitors, or a combination of both.

\section{Materials and methods}

Cell culture and drugs. Four lung adenocarcinoma cell lines (RERF-LC-KJ, HCC827, NCI-H1650 and NCI-H1975) were used in this study and were obtained as previously described (5). HCC827 and H1650 cells harbor deletion mutations in exon 19 (del E746-A750), and H1975 cells contain two-point mutations of T790M in exon 20, which confers resistance to EGFR kinase inhibitors and L858R in exon 21 in the EGFR gene (13). HCC827 cells are sensitive to EGFR inhibitors whereas $\mathrm{H} 1650$ cells are resistant to the drugs, probably because of the loss of PTEN (13). LC-KJ cells have neither EGFR nor K-ras gene mutation (5). Of these four cell lines, HCC 827 cells are also sensitive to Src inhibitors whereas the other three cell lines are relatively resistant to these inhibitors $(5,14)$. All cell lines were maintained in RPMI-1640 medium supplemented with $10 \%$ heat-inactivated fetal bovine serum, $50 \mathrm{U} / \mathrm{ml}$ penicillin and $50 \mu \mathrm{g} / \mathrm{ml}$ streptomycin. Cells were grown to subconfluence in $94-\mathrm{mm}$ tissue culture dishes (Greiner Bio-One, Tokyo, Japan) and were then trypsinized. The cells were then seeded on 90-mm low cell binding dishes (Nalge Nunc International KK, Tokyo, Japan) for suspension culture or the above-mentioned tissue culture dishes for monolayer culture, and were then cultured at $37^{\circ} \mathrm{C}$ in a humidified incubator with $5 \% \mathrm{CO}_{2}$ in air. The cells cultured on low cell binding or conventional culture dishes for $48 \mathrm{~h}$ were then treated with the small-molecule inhibitor(s), as described below, for another $24 \mathrm{~h}$. As a control, cells were treated with the same concentration of dimethyl sulfoxide (DMSO) (Sigma-Aldrich, St. Louis, MO, USA). The drugs used in this study were the Bcl-2 inhibitor ABT-263 (Toronto Research Chemicals, North York, Canada), two Src family tyrosine kinase inhibitors, bosutinib (SKI-606) (Symansis, Auckland, New Zealand) or PP1 (Invitrogen, Carlsbad, CA,
USA), and the Abl inhibitor imatinib (Toronto Research Chemicals). Stock solutions of these drugs were prepared at $10 \mathrm{mmol} / \mathrm{l}(\mathrm{mM})$ in DMSO.

Western blotting. All cell lines were maintained on either low cell binding or conventional dishes for $72 \mathrm{~h}$. The cells were then lysed in NuPAGE LDS sample buffer (Invitrogen). The whole cell lysates were subjected to SDS-PAGE (NuPAGE 4-12\% Bis-Tris Gel; Invitrogen) followed by blotting with indicated antibodies, and detected by Supersignal West Pico chemiluminescent substrate (Thermo Scientific, Rockford, IL, USA). The antibodies used in this study were as follows: anti-PARP-1 (p116/p85) (E78; 1:500 dilution; Epitomics, Burlingame, CA, USA), anti-Src (36D10; 1:1,000 dilution; Cell Signaling Technology Japan, Tokyo, Japan), antiphospho-Src Family (Tyr416) (1:250; Cell Signaling Technology Japan), anti-Mcl-1 (S-19; 1:500 dilution; Santa Cruz Biotechnology, Santa Cruz, CA, USA), anti-Bcl-X (S-18; 1:500 dilution; Santa Cruz Biotechnology), anti-Bim (Y36; 1:500 dilution; Epitomics), and anti- 3 -actin (AC-15; 1:5,000 dilution; Sigma-Aldrich). To evaluate the levels of apoptosis, the band intensity of the pro- and cleaved-forms of poly (ADP-ribose) polymerase (PARP)-1 was measured on an X-ray film using Image $\mathrm{J}$ software. PARP-1 is considered to be an intracellular death substrate and its cleaved form is used as a representative marker of apoptosis (15).

Preparation of cell blocks. Cell blocks of the four suspension cell lines cultured for $72 \mathrm{~h}$ were made as previously described (16). The cell blocks embedded in agarose were fixed in formalin and then embedded in paraffin. Thin sections ( $3 \mu \mathrm{m}$ thick) were stained with hematoxylin and eosin (HE) and immunocytochemical staining of phosphorylated-Src ( $p-\mathrm{Src})$ and cleaved caspase-3 was performed as previously described (5). We counted at least 1,000 cells for the apoptotic bodies/ 100 cells in each cell line to examine whether there were differences in the number of apoptotic bodies (pyknotic nuclear fragments of apoptotic cells) between tumor spheroids formed upon detachment and treated with ABT-263, PP1, both or neither. We also evaluated whether p-Src or cleaved caspase-3 was expressed in spheroids treated with or without the inhibitor(s). Membranous staining of $\mathrm{p}$-Src was scored based on intensity ( 0 , none; 1 , weak; 2 , intermediate; 3 , strong) and proportion of tumor cells expressing the marker (0, none; $1,<1 / 100 ; 2,1 / 100-1 / 10 ; 3,1 / 10-1 / 3 ; 4,1 / 3-2 / 3 ; 5$, $>2 / 3$ ) using the Allred scoring method (17).

Statistical analysis. Differences in rates of apoptosis between cells treated with ABT-263, Src inhibitor, both, or neither were evaluated by paired t-tests. P-values $<0.05$ were considered significant. All statistical calculations were performed with the JMP software system (JMP for Windows version 7; SAS Institute Japan; Tokyo, Japan).

\section{Results}

The Bcl-2 inhibitor ABT-263 significantly enhances the degree of anoikis in lung adenocarcinoma cells treated with the Src inhibitor bosutinib. Combination therapy with ABT263 and bosutinib effectively induced anoikis in all cell lines 

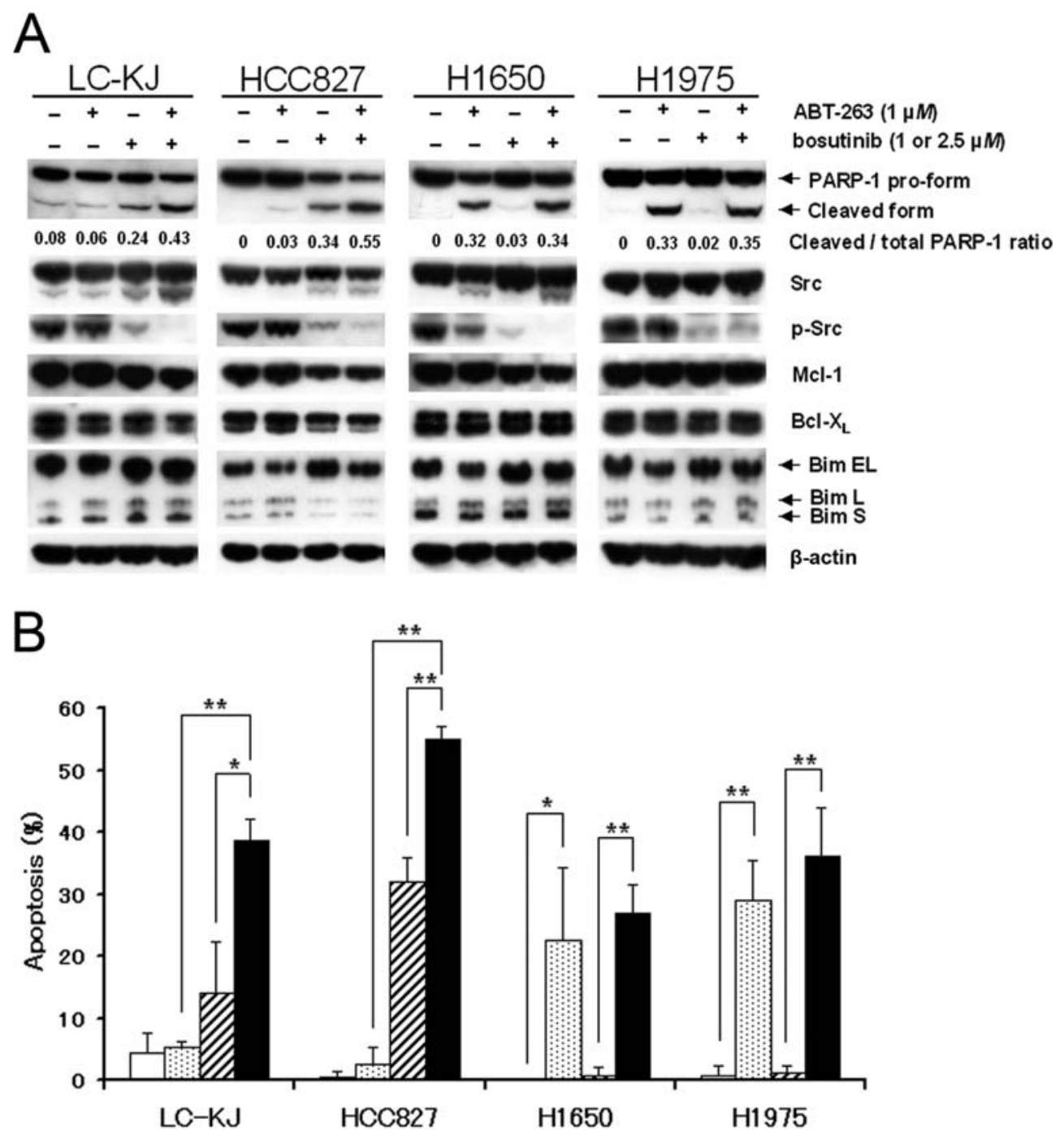

control $\mathrm{F}$ ABT-263 $\boldsymbol{Z}$ bosutinib $\mathrm{ABT}-263+$ bosutinib

Figure 1. ABT-263 and bosutinib have synergistic effects on cell death of LC-KJ and HCC827 cells, and suspension H1650 and H1975 cells are sensitive to ABT-263. (A) Western blotting to examine the effects of ABT-263, bosutinib, both, or neither in LC-KJ, HCC827, H1650 and H1975 cells. Cells were treated with bosutinib (LC-KJ, H1650 and H1975, $2.5 \mu \mathrm{M}$ bosutinib; HCC827, $1 \mu \mathrm{M}$ bosutinib) in the presence or absence of $1 \mu \mathrm{M}$ ABT-263 for $24 \mathrm{~h}$. The cleaved/total PARP-1 ratio determined for each cell line using Image J software is also shown. (B) Apoptosis was assessed by PARP-1 cleavage. Values are the means + standard deviation (SD) of three (LC-KJ and HCC827) or four (H1650 and H1975) independent experiments. ${ }^{*} \mathrm{P}<0.05 ;{ }^{* *} \mathrm{P}<0.01$.

examined (Fig. 1A and B). This was probably due to the proapoptotic effect of Bim, which was induced in the presence of bosutinib in each cell line, the addition of ABT-263, and the levels of other $\mathrm{BH} 3$-only proteins exceeded the antiapoptotic capacity of $\mathrm{Mcl}-1, \mathrm{Bcl}-\mathrm{X}_{\mathrm{L}}$, and other anti-apoptotic Bcl-2 family members (Fig. 1A). In LC-KJ cells, the extent of anoikis induced by combination therapy was markedly greater than that induced by ABT-263 or bosutinib alone (combination vs. bosutinib alone: 38.7 vs. $14.0 \%, \mathrm{P}=0.0134$; combination vs. ABT-263 alone: 38.7 vs. $5.3 \%, \mathrm{P}=0.0021$ ) (Fig. 1B). In HCC827 cells, although bosutinib ( $1 \mu \mathrm{M})$ alone substantially induced anoikis (bosutinib alone versus DMSO: 31.9 vs. $0.5 \%, P=0.0040)$, the combination therapy was significantly more effective than ABT-263 or bosutinib alone (combination vs. bosutinib alone: 55.0 vs. $31.9 \%, \mathrm{P}=0.0030$; combination vs. ABT-263: 55.0 vs. 2.6\%, P<0.0001) (Fig. 1B). On the other hand, ABT-263 $(1 \mu \mathrm{M})$ alone significantly induced anoikis and seemed to reduce the expression of $\mathrm{p}-\mathrm{Src}$ in suspension H1650 cells (ABT-263 vs. DMSO: 22.3 vs. $0.0 \%, \mathrm{P}=0.0290$ ) (Fig. 1A and B). Nevertheless the degree of anoikis in H1650 cells treated with the combination was greatest, and was significantly greater than bosutinib alone (combination vs. bosutinib alone: 27.2 vs. $0.7 \%, \mathrm{P}=0.0007$ ). However, there was no marked difference in anoikis induction between combination therapy and ABT-263 alone (combination vs. ABT-263 alone: 27.2 vs. $22.3 \%, \mathrm{P}=0.4216$ ). In H1975 cells, ABT-263 also markedly induced anoikis (ABT-263 alone versus DMSO: 28.9 vs. $0.7 \%, \mathrm{P}=0.0053$ ) (Fig. 1A and B). The extent of anoikis induced by combination therapy was also greatest in H1975 cells, and combination therapy was significantly more efficacious than bosutinib alone (combination vs. bosutinib alone: 36.2 vs. $1.1 \%$, $\mathrm{P}=0.0038$ ). However, there was no significant difference in anoikis induction between combination therapy and 
A

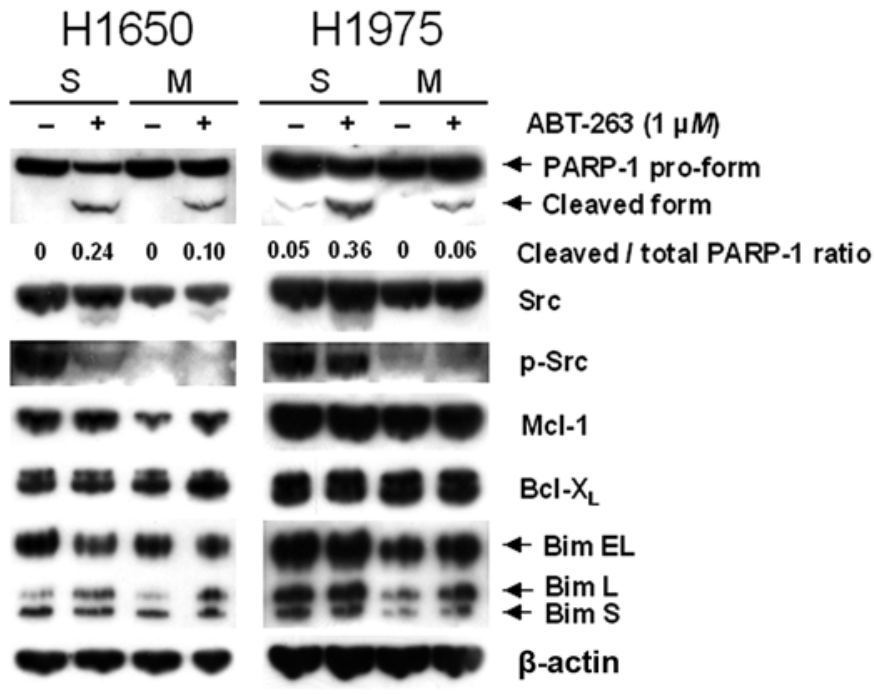

B

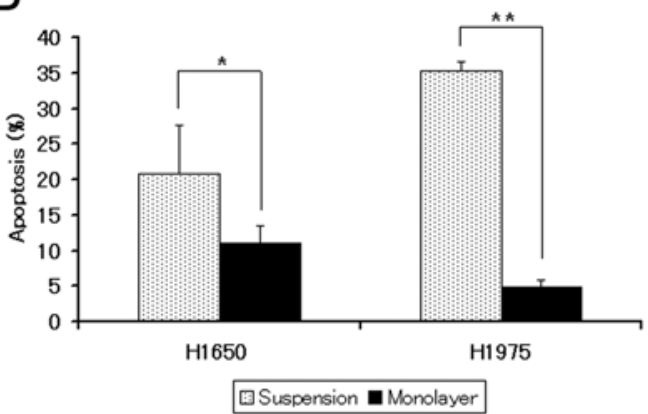

C

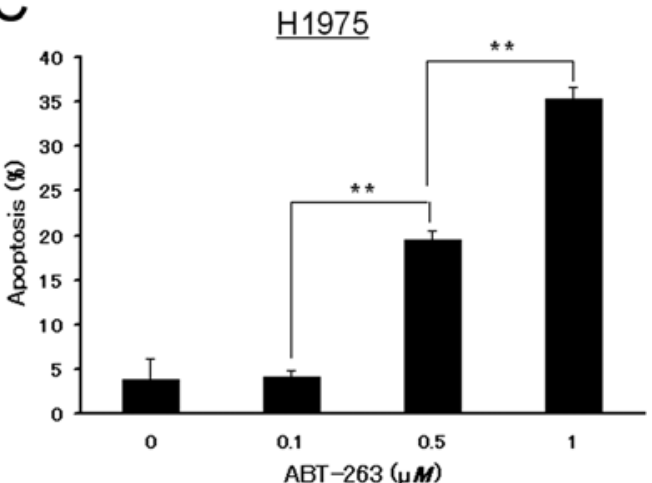

Figure 2. The levels of apoptosis induced by treatment with ABT-263 in monolayer culture are lower than those in suspension culture. (A) Western blotting to examine the effects of ABT-263 suspension H1650 and H1975 cells or monolayer culture. Cells were treated with ABT-263 (1 $\mu$ M) for 24 h. The cleaved/total PARP-1 ratio determined for each cell line using Image J software is also represented. S, suspension culture; M, monolayer culture. (B) Apoptosis was assessed by PARP-1 cleavage. Values are the means $+\mathrm{SD}$ of three $(\mathrm{H} 1975)$ or four $(\mathrm{H} 1650)$ independent experiments. ${ }^{*} \mathrm{P}<0.05$; ${ }^{* *} \mathrm{P}<0.01 .(\mathrm{C})$ Suspension H1975 cells were treated with different concentrations of ABT-263 for $24 \mathrm{~h}$. Apoptosis was assessed by PARP-1 cleavage. Values are the means $+\mathrm{SD}$ of three independent experiments. ${ }^{* *} \mathrm{P}<0.01$.

ABT-263 alone (combination vs. ABT-263 alone: $36.2 \%$ vs. $28.9 \%, \mathrm{P}=0.1285)$. These findings indicate synergistic effects of ABT-263 and bosutinib in LC-KJ and HCC827 cells. Unexpectedly, ABT-263 alone cause marked anoikis in H1650 and H1975 cells in the suspension culture system.

ABT-263 is much less effective in inducing apoptosis in monolayer culture than in suspension. The degree of apoptosis induced by ABT-263 in H1650 or H1975 cells upon attachment was much smaller than that upon detachment (Fig. 2A and B). The amount of Bim, which was induced by the suspension condition, might affect the commitment to apoptosis. In H1650 cells, although ABT-263 (1 $\mu \mathrm{M})$ significantly induced apoptosis in the monolayer and suspension culture conditions compared with control (ABT-263 vs. DMSO: monolayer, 11.0 vs. $0.0 \%, \mathrm{P}=0.0031$; suspension, 20.8 vs. $0.0 \%, \mathrm{P}=0.0091$ ), the extent of apoptosis upon attachment was much smaller than that upon detachment (monolayer versus suspension: 11.0 vs. $20.8 \%, \mathrm{P}=0.0376$ ) (Fig. 2B). The expression of p-Src in H1650 cells upon detachment was decreased in the presence of ABT-263. In H1975 cells, although $1 \mu \mathrm{M}$ ABT-263 significantly induced apoptosis in the monolayer and suspension culture conditions compared with control (ABT-263 vs. DMSO: monolayer, 4.8 vs. $0.0 \%, \mathrm{P}=0.0176$; suspension, 35.3 vs. $3.8 \%, \mathrm{P}=0.0033$ ), the extent of apoptosis upon attachment was significantly smaller than that upon detachment (monolayer vs. suspension: 4.8 vs. $35.3 \%, \mathrm{P}=0.0001$ ) (Fig. $2 \mathrm{~B}$ ). We also confirmed that the levels of anoikis in H1975 cells treated with ABT-263 upon detachment were induced in a concentration-dependent manner ( 1 vs. $0.5 \mu \mathrm{M}$ : 35.3 vs. $19.5 \%, \mathrm{P}=0.0021 ; 0.5$ vs. $0.1 \mu \mathrm{M}: 19.5$ vs. $4.1 \%, \mathrm{P}=0.0006 ; 0.1 \mu \mathrm{M}$ vs. DMSO: 4.1 vs. $3.8 \%, \mathrm{P}=0.9011$ ) (Fig. 2C).

ABT-263 significantly enhances the degree of anoikis in lung adenocarcinoma cells treated with another Src inhibitor, PP1. Combination therapy with ABT-263 markedly enhanced the anoikis levels in all cell lines treated with PP1, another Src kinase inhibitor in LC-KJ (combination vs. PP1: 17.0 vs. $4.7 \%, \mathrm{P}=0.0126$ ), HCC827 (31.7 vs. $12.0 \%$, respectively, $\mathrm{P}=0.0111$ ), $\mathrm{H} 1650$ (60.0 vs. $4.0 \%$, respectively, $\mathrm{P}=0.0310$ ), and $\mathrm{H} 1975$ cells (41.8 vs. $1.6 \%$, respectively, $\mathrm{P}=0.0077$ ) (Fig. 3A and B). Although PP1 and bosutinib are known to inhibit the kinase activity of the Abl as well as Src $(18,19)$, imatinib, a potent Abl inhibitor, did not affect the levels of anoikis at concentrations up to $10 \mu \mathrm{M}$ (Fig. 3A and B). The levels of anoikis induced by imatinib were almost identical with those observed in the control cells (Figs. 1A and 3A). These results suggest that Abl does not play a crucial role in anoikis resistance in lung adenocarcinoma cells.

Confirmation of the pro-apoptotic effects of combination treatment with ABT-263 and PPI in terms of the number of apoptotic bodies counted on cell blocks. The number of apoptotic bodies counted in each cell line treated with ABT-263, PP1, both, or neither, showed similar results to those deter- 
A

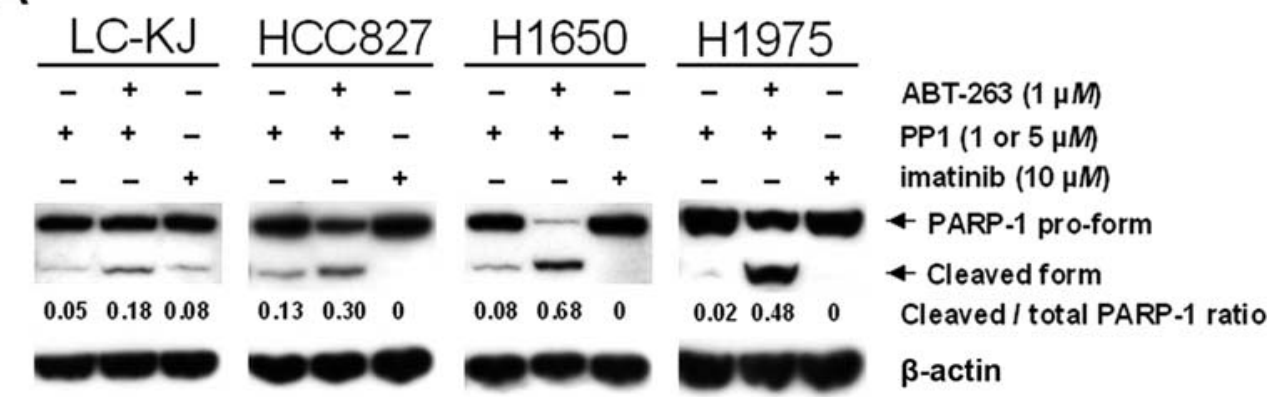

B

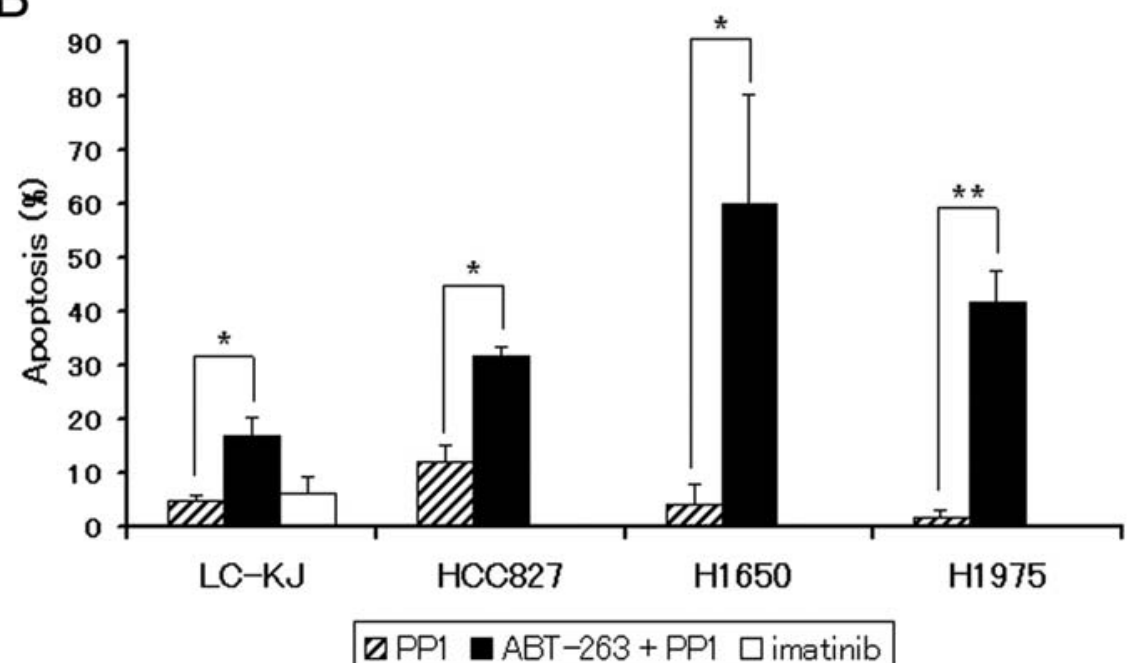

Figure 3. ABT-263 enhances the susceptibility of lung adenocarcinoma cells treated with PP1 to anoikis. (A) Western blotting to examine the effects of ABT-263, PP1, a combination of PP1 and ABT-263, or imatinib, on apoptosis (anoikis) in LC-KJ, HCC827, H1650 and H1975 cells. Cells were treated with PP1 (LC-KJ, $\mathrm{H} 1650$ and $\mathrm{H} 1975,5 \mu \mathrm{M}$ PP1; HCC827, $1 \mu \mathrm{M} \mathrm{PP} 1)$ in the presence or absence of $1 \mu \mathrm{M}$ ABT-263 or $10 \mu \mathrm{M}$ imatinib for $24 \mathrm{~h}$. The cleaved/total PARP-1 ratio determined for each cell line using Image $\mathbf{J}$ software is also shown. B-actin is shown as a loading control. (B) Apoptosis was assessed in terms of PARP-1 cleavage. Values are the means $+\mathrm{SD}$ of three independent experiments. ${ }^{*} \mathrm{P}<0.05 ;{ }^{* *} \mathrm{P}<0.01$.

mined by the PARP-1 cleavage assay (Figs. 1B and 4A). The expression of cleaved caspase-3, another apoptosis marker, was observed in proportion to the number of apoptotic bodies (Fig. 4B). ABT-263 effectively induced anoikis in H1650 and $\mathrm{H} 1975$ cells that maintained their expression of $\mathrm{p}-\mathrm{Src}$ (Fig. 4A and B). However, the membranous expression of p-Src in H1650 cells treated with ABT-263 alone was slightly decreased compared with that in control cells (Fig. 4B).

\section{Discussion}

Here, we have shown that the Bcl-2 inhibitor ABT-263 affects the survival of four suspension lung adenocarcinoma cell lines cultured. Interestingly, the four cell lines varied in their sensitivity to $1 \mu \mathrm{M}$ ABT-263. We did not observe a significant increase in anoikis in LC-KJ or HCC827 cells treated with ABT-263 alone whereas H1650 and H1975 cells upon detachment were very sensitive to ABT-263 and readily underwent anoikis in the presence of ABT-263. It seems unlikely that the varying susceptibility to ABT-263 depends on the expression levels of Mcl-1, to which ABT-263 can hardly bind to suppress anti-apoptotic activity $(7,10)$, because Mcl-1 was expressed in all suspension cell lines. Decreased expression levels of $\mathrm{p}$-Src might play a role in anoikis induction in H1650 cells treated with ABT-263. However, the molecular mechanisms underlying the varying sensitivity to ABT-263 remains to be solved. We also observed that the pro-apoptotic effect of ABT-263 on H1650 or H1975 cells upon detachment was substantially greater than that upon attachment. The levels of apoptosis induced by ABT-263 $(1 \mu \mathrm{M})$ in $\mathrm{H} 1650$ or $\mathrm{H} 1975$ cells upon attachment in this study were consistent with those induced by ABT-737 $(1 \mu \mathrm{M})$ in the same cells in an earlier report (11). In LC-KJ and HCC827 cells, the extent of anoikis induced by combination treatment with ABT-263 and an Src inhibitor (bosutinib or PP1) was significantly greater than that induced by each agent alone, demonstrating synergistic activity of the two inhibitors. Indeed, combination therapy was more effective than each agent alone in H1650 and H1975 cells, although the synergistic effect was not always apparent in these cells.

Several phase II clinical trials with Src kinase inhibitors (dasatinib or AZD0530) in patients with NSCLC are currently underway $(20,21)$. Since the kinase activity of Src promotes growth, invasion, angiogenesis and metastasis of NSCLCs (22), Src inhibitors can probably improve the outcome of patients with NSCLC to some extent. However, we have 

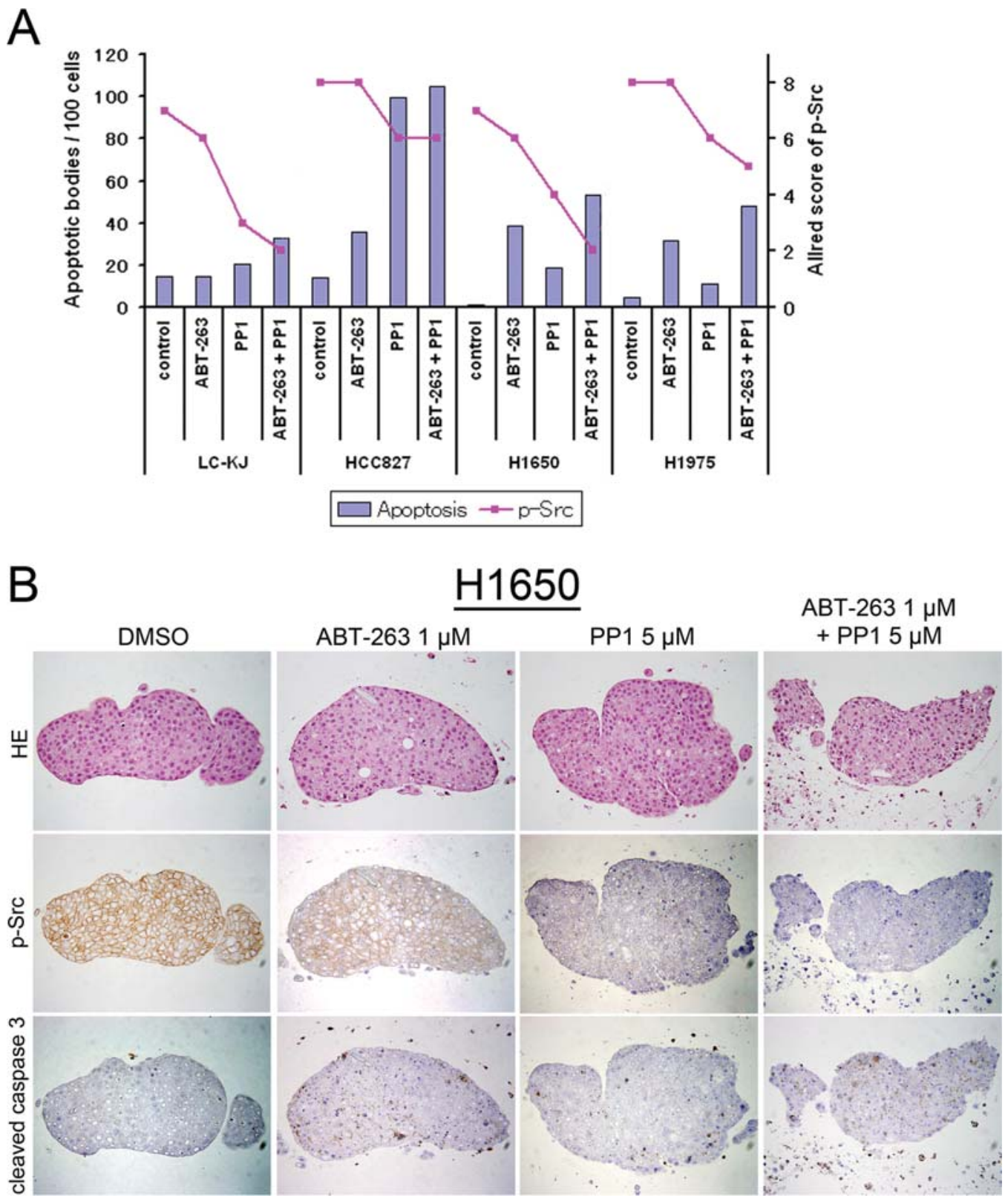

Figure 4. Combination treatment with ABT-263 and PP1 induces anoikis in lung adenocarcinoma cell lines. (A) The number of apoptotic bodies/100 cells was counted, and Allred scores for p-Src were evaluated in each cell line. (B) Representative images of cell blocks of suspension $\mathrm{H} 1650$ cells cultured for $72 \mathrm{~h}$ are shown. Cells were treated with $1 \mu \mathrm{M}$ ABT-263, $5 \mu \mathrm{M}$ PP1, both, or neither for $24 \mathrm{~h}$.

already observed that the Src kinase activity of lung adenocarcinoma cells is much greater in floating conditions than in the ECM-adherent state (5). Although not all of the suspension lung adenocarcinoma cell lines examined were very sensitive to Src inhibitors, as observed in these cell lines treated with ABT-263, combination therapy with an Src inhibitor (bosutinib or PP1) and ABT-263 effectively induced anoikis in the four cell lines examined upon detachment. Therefore, we expect that combination therapy with ABT-263 and Src inhibitor as components of adjuvant chemotherapy for patients with vessel invasion-positive lung adenocarcinoma may induce anoikis in some intravessel floating tumor cells and consequently reduce the development of metastasis. However, further studies, including in vivo animal studies, are still needed because a small fraction of tumor cells, termed drug-tolerant persisters, could survive and proliferate even during combination treatment, as reported in gefitinibtreated PC-9 cells, a gefitinib-sensitive lung adenocarcinoma cell line (23).

In summary, two suspension lung adenocarcinoma cell lines ( $\mathrm{LC}-\mathrm{KJ}$ and $\mathrm{HCC} 827$ cells) were very sensitive to combination therapy with a Bcl-2 inhibitor (ABT-263) and an Src inhibitor (bosutinib or PP1), and underwent anoikis. Furthermore, these two drugs exerted synergistic effects in both cell lines. Although combination therapy also induced anoikis in the other cell lines (H1650 and H1975 cells) cultured upon detachment, both types of cells underwent significant anoikis in the presence of ABT-263 $(1 \mu \mathrm{M})$ alone. We observed that the four cell lines showed varying sensitivity to ABT-263 and the Src inhibitors, which may 
reflect the diversity of lung adenocarcinomas. However, combination therapy had the greatest effect on anoikis in all the cell lines examined here. These findings suggest that combination therapy with ABT-263 and an Src inhibitor could induce anoikis in intravessel floating lung adenocarcinoma cells and may reduce the development of distant metastases in patients with vessel invasion-positive lung adenocarcinoma.

\section{Acknowledgements}

This study was supported in part by a Grant-in-Aid for Young Scientists (B) (20790294 and 22790367) from the Ministry of Education, Culture, Sports, Science and Technology of Japan (Y. Sakuma).

\section{References}

1. Sakuma Y, Okamoto N, Saito H, et al: A logistic regression predictive model and the outcome of patients with resected lung adenocarcinoma of $2 \mathrm{~cm}$ or less in size. Lung Cancer 65: 85-90, 2009.

2. Frisch SM and Francis H: Disruption of epithelial cell-matrix interactions induces apoptosis. J Cell Biol 124: 619-626, 1994.

3. Wei L, Yang Y, Zhang X and Yu Q: Altered regulation of Src upon cell detachment protects human lung adenocarcinoma cells from anoikis. Oncogene 23: 9052-9061, 2004.

4. Uekita T, Jia L, Narisawa-Saito M, Yokota J, Kiyono T and Sakai R: CUB domain-containing protein 1 is a novel regulator of anoikis resistance in lung adenocarcinoma. Mol Cell Biol 27: 7649-7660, 2007.

5. Sakuma Y, Takeuchi T, Nakamura Y, et al: Lung adenocarcinoma cells floating in lymphatic vessels resist anoikis by expressing phosphorylated Src. J Pathol 220: 574-585, 2010.

6. Cragg MS, Harris C, Strasser A and Scott CL: Unleashing the power of inhibitors of oncogenic kinases through $\mathrm{BH} 3$ mimetics. Nat Rev Cancer 9: 321-326, 2009.

7. Vogler M, Dinsdale D, Dyer MJ and Cohen GM: Bcl-2 inhibitors: small molecules with a big impact on cancer therapy. Cell Death Differ 16: 360-367, 2009.

8. Willis SN, Fletcher JI, Kaufmann T, et al: Apoptosis initiated when BH3 ligands engage multiple $\mathrm{Bcl}-2$ homologs, not $\mathrm{Bax}$ or Bak. Science 315: 856-859, 2007.

9. Oltersdorf T, Elmore SW, Shoemaker AR, et al: An inhibitor of Bcl-2 family proteins induces regression of solid tumours. Nature 435: 677-681, 2005.
10. Tse C, Shoemaker AR, Adickes J, et al: ABT-263: a potent and orally bioavailable Bcl-2 family inhibitor. Cancer Res 68: 3421-3428, 2008.

11. Cragg MS, Kuroda J, Puthalakath H, Huang DC and Strasser A: Gefitinib-induced killing of NSCLC cell lines expressing mutant EGFR requires BIM and can be enhanced by $\mathrm{BH} 3$ mimetics. PLoS Med 4: 1681-1689, 2007.

12. Gong Y, Somwar R, Politi K, Balak M, Chmielecki J, Jiang X and $\mathrm{Pao} \mathrm{W}$ : Induction of BIM is essential for apoptosis triggered by EGFR kinase inhibitors in mutant EGFR-dependent lung adenocarcinomas. PLoS Med 4: e294, 2007.

13. Sharma SV, Bell DW, Settleman J and Haber DA: Epidermal growth factor receptor mutations in lung cancer. Nat Rev Cancer 7: 169-181, 2007.

14. Zhang J, Kalyankrishna S, Wislez M, et al: SRC-family kinases are activated in non-small cell lung cancer and promote the survival of epidermal growth factor receptor-dependent cell lines. Am J Pathol 170: 366-376, 2007.

15. Soldatenkov VA and Smulson M: Poly(ADP-ribose) polymerase in DNA damage-response pathway: implications for radiation oncology. Int J Cancer 90: 59-67, 2000.

16. Mansy SS: Agarose cell block: innovated technique for the processing of urine cytology for electron microscopy examination. Ultrastruct Pathol 28: 15-21, 2004.

17. Harvey JM, Clark GM, Osborne CK and Allred DC: Estrogen receptor status by immunohistochemistry is superior to the ligand-binding assay for predicting response to adjuvant endocrine therapy in breast cancer. J Clin Oncol 17: 1474-1481, 1999.

18. Tatton L, Morley GM, Chopra R and Khwaja A: The Srcselective kinase inhibitor PP1 also inhibits Kit and Bcr-Abl tyrosine kinases. J Biol Chem 278: 4847-4853, 2003.

19. Golas JM, Arndt K, Etienne C, et al: SKI-606, a 4-anilino-3quinolinecarbonitrile dual inhibitor of Src and Abl kinases, is a potent antiproliferative agent against chronic myelogenous leukemia cells in culture and causes regression of K562 xenografts in nude mice. Cancer Res 63: 375-381, 2003.

20. Kim LC, Song L and Haura EB: Src kinases as therapeutic targets for cancer. Nat Rev Clin Oncol 6: 587-595, 2009.

21. Haura EB, Tanvetyanon T, Chiappori A, et al: Phase I/II study of the Src inhibitor dasatinib in combination with erlotinib in advanced non-small-cell lung cancer. J Clin Oncol 28: 1387-1394, 2010.

22. Giaccone G and Zucali PA: Src as a potential therapeutic target in non-small-cell lung cancer. Ann Oncol 19: 1219-1223, 2008.

23. Sharma SV, Lee DY, Li B, et al: A chromatin-mediated reversible drug-tolerant state in cancer cell subpopulations. Cell 141: 69-80, 2010. 